\title{
A new phase of activity of the Herbig Be star HD 200775 in 2001: Evidence for binarity ${ }^{\star}$
}

\author{
M. A. Pogodin ${ }^{1,2}$, A. S. Miroshnichenko ${ }^{1,3}$, A. E. Tarasov ${ }^{4,5}$, M. P. Mitskevich ${ }^{4,5}$, G. A. Chountonov ${ }^{6}$, \\ V. G. Klochkova ${ }^{6}$, M. V. Yushkin ${ }^{6}$, N. Manset $^{7}$, K. S. Bjorkman ${ }^{3}$, N. D. Morrison ${ }^{3}$, and J. P. Wisniewski ${ }^{3}$ \\ 1 Pulkovo Observatory, Saint-Petersburg 196140, Russia \\ ${ }^{2}$ Isaac Newton Institute of Chile, Saint-Petersburg Branch, Russia \\ ${ }^{3}$ Ritter Observatory, Dept. of Physics \& Astronomy, The University of Toledo, Toledo, OH 43606-3390, USA \\ 4 Crimean Astrophysical Observatory, Nauchny, Crimea 334413, Ukraine \\ 5 Isaak Newton Institute of Chile, Crimean Branch, Ukraine \\ ${ }^{6}$ Special Astrophysical Observatory at Northern Caucasus, Nizhnij Arkhys 357147, Russia \\ 7 CFHT Corporation, 65-1238 Mamalahoa Hwy, Kamuela, HI 96743, Hawaii, USA
}

Received 6 June 2003 / Accepted 18 December 2003

\begin{abstract}
The results of high-resolution spectroscopy of the Herbig Be star HD 200775 obtained within the framework of a cooperative observing programme in 2000-2002 are presented. A new high-activity phase of the object's $\mathrm{H} \alpha$ line occurred in the middle of 2001 in full agreement with a 3.68-year periodicity predicted by Miroshnichenko et al. (1998). A complicated picture of the $\mathrm{H} \alpha$ line profile variability near the activity maximum phase turned out to be very similar to that observed during the previous one in 1997. Variations of the radial velocity with the activity phase are detected in He I, Si II, and S II photospheric lines. The observed phenomena are interpreted in the framework of a model in which the star, together with its gaseous envelope, is a component of an eccentric binary system. A preliminary orbital solution is derived, and the system's parameters are estimated from the radial velocity curves of the $\mathrm{H} \alpha$ emission line. We find that the orbital eccentricity is $e \sim 0.3$, the mean companion separation is $\sim 1000 R_{\odot}$, and the secondary companion is most likely to be a $\sim 3.5 M_{\odot}$ pre-main sequence object. We emphasize the importance of coordinated spectroscopic and interferometric observations at different phases of the object's activity for further understanding the properties of the system.
\end{abstract}

Key words. techniques: spectroscopic - stars: circumstellar matter - stars: individual: HD 200775 -

stars: binaries: spectroscopic - stars: pre-main sequence

\section{Introduction}

In this paper we present further results of a long-term cooperative programme of spectroscopic investigation of the bright northern Herbig Be star HD 200775, which demonstrates significant spectral variations. The detailed observational background for the object and the previous results of the programme have been reported by Beskrovnaya et al. (1994), Miroshnichenko et al. (1998), Pogodin et al. (2000), and in a number of papers referenced therein.

The most significant manifestation of the object's activity is seen in the $\mathrm{H} \alpha$ and $\mathrm{H} \beta$ emission lines. When the object is in a low state, the emission Balmer lines show double-peaked profiles. During active periods, the line emission becomes

Send offprint requests to: M. A. Pogodin,

e-mail: pogodin@gao.spb.ru

* Partially based on observations collected at the Canada-FranceHawaii telescope (CFHT), operated by the National Research Council of Canada, the Centre National de la Recherche Scientifique, and University of Hawaii. stronger and a complicated multicomponent structure of the profiles develops. Miroshnichenko et al. (1998) argue that appearance of the active states has a cyclic character with a period $P=1345$ days (3.68 years). The most detailed analysis of the spectroscopic behaviour of HD 200775 around its last maximum activity in 1997 was carried out by Pogodin et al. (2000). They found that the onset of the high-activity phase of $\mathrm{H} \alpha$ and $\mathrm{H} \beta$ was characterized by: a) a significant rise of the equivalent width $(E W)$; and b) a doubling of the central absorption feature with a new, blueshifted component appearing in addition to the pre-existing redshifted one with a larger positive radial velocity $(R V)$ and a smaller depth. It was suggested that the active phenomena in the spectral behaviour of HD 200775 are connected with the generation of a strong stellar wind, interacting with the circumstellar (CS) disk. However, no specific mechanism for the wind generation was proposed. An alternative hypothesis that the cyclic activity of HD 200775 is a result of its binarity was suggested also, but it was noted that further observational support was needed to verify it. 
In this paper, high-resolution spectroscopic data obtained in 2000-2002 are analysed. The previously estimated period of $P=3.68$ years is verified, the binary hypothesis is considered, and orbital elements are estimated. The binary hypothesis for the system is thus validated.

In Sect. 4.2 we discuss the results by Ismailov (2003), who has obtained a different orbital solution for the system.

\section{Observations}

Our new run of high-resolution spectroscopic observations of HD 200775 was carried out between September 2000 and September 2002 using a number of instruments at different observatories.

At the Crimean Astrophysical Observatory (CrAO, Ukraine), the coudé spectrograph with a CCD detector (SDS-9000 "Photometric GmbH", a spectral resolving power $R \sim 30000$ ) at the $2.6 \mathrm{~m}$ Shajn telescope was used. The data reduction followed standard procedures and was done with the SPE code developed by Sergeev.

At Ritter Observatory (Toledo, USA) a $1 \mathrm{~m}$ telescope equipped with a Wright Instruments Ltd.CCD camera was employed. The échelle spectra consisted of 9 non-overlapping $\sim 70 \AA$ orders with a $R \simeq 26000$. Several spectra were obtained at the $2.1 \mathrm{~m}$ Otto Struve telescope of the McDonald Observatory (Mt. Locke, Texas, USA) with the Sandiford échelle-spectrometer (McCarthy et al. 1993) and $R \sim 60000$. A $1200 \times 400$ pixel CCD was used. These data were reduced with IRAF ${ }^{1}$.

At the $6 \mathrm{~m}$ telescope of the Special Astrophysical Observatory of the Russian Academy of Sciences at Northern Caucasus (SAO, Russia) we used several CCD equipped spectrographs: the Main Stellar Spectrograph (MSS) with $R=15000$, PFES with $R=15000$ (Panchuk et al. 1998), LYNX with $R=30000$ (Panchuk et al. 1999a), and NES with $R=60000$ (Panchuk et al. 1999b).

At the 3.6-m CFHT (Hawaii, USA), the high-resolution $(R \sim 100000)$ Gecko échelle spectrograph, fiber-fed from the Cassegrain focus (Baudrand \& Vitry 2000), and a $2048 \times$ $4608 \times 13.5 \mu \mathrm{m}^{2}$ thinned back-illuminated EEV chip were used. The fiber was continuously agitated to overcome modal noise (Baudrand \& Walker 2001). Narrow spectral regions near $\mathrm{H} \delta$ and $\mathrm{H} \alpha(\Delta \lambda \sim 60 \AA)$ were observed. The SAO were reduced with MIDAS, while the CFHT data were reduced with IRAF.

Table 1 lists the observations performed in the $\mathrm{H} \alpha$ region, and Table 2 lists spectra that were obtained in regions containing photospheric lines. Table 2 also contains information about a few spectra from our previous observing run in 1994-1999 (Pogodin et al. 2000).

The continuum signal-to-noise ratio $(S / N)$ in all the spectra varies between 50 and 200 depending on the telescope size and

${ }^{1}$ IRAF is distributed by the National Optical Astronomy Observatories, which are operated by the Association of Universities for Research in Astronomy, Inc., under contract with the National Science Foundation.
Table 1. Summary of the H $\alpha$ observations of HD 200775.

\begin{tabular}{|c|c|c|c|c|c|}
\hline Date & $\begin{array}{c}\text { HJD } \\
2400000+\end{array}$ & Phase & $N$ & $\begin{array}{r}E W \\
\AA\end{array}$ & Observatory \\
\hline 16.09 .00 & 51803.763 & 0.846 & 1 & 73.2 & Ritter \\
\hline 18.09 .00 & 51805.618 & 0.847 & 1 & 70.2 & Ritter \\
\hline 11.10 .00 & 51828.573 & 0.864 & 1 & 84.3 & Ritter \\
\hline 12.10 .00 & 51829.589 & 0.865 & 1 & 82.3 & Ritter \\
\hline 13.10 .00 & 51830.584 & 0.866 & 1 & 76.2 & Ritter \\
\hline 13.10 .00 & 51830.600 & 0.866 & 11 & 78.9 & SAO \\
\hline 14.10 .00 & 51831.560 & 0.867 & 10 & 86.1 & SAO \\
\hline 15.10 .00 & 51832.590 & 0.867 & 8 & 82.4 & SAO \\
\hline 19.10 .00 & 51836.583 & 0.870 & 1 & 84.3 & Ritter \\
\hline 28.10 .00 & 51845.517 & 0.877 & 1 & 81.3 & Ritter \\
\hline 29.10 .00 & 51846.579 & 0.878 & 1 & 84.2 & Ritter \\
\hline 01.11 .00 & 51849.511 & 0.880 & 1 & 81.9 & Ritter \\
\hline 05.11 .00 & 51853.614 & 0.883 & 1 & 80.5 & Ritter \\
\hline 04.12 .00 & 51883.462 & 0.905 & 1 & 85.9 & Ritter \\
\hline 18.03.01 & 51986.969 & 0.982 & 1 & 87.7 & Ritter \\
\hline 19.03.01 & 51987.965 & 0.983 & 1 & 92.0 & Ritter \\
\hline 28.03.01 & 51996.909 & 0.990 & 1 & 94.5 & Ritter \\
\hline 14.04 .01 & 52013.880 & 0.002 & 1 & 98.4 & SAO \\
\hline 28.04 .01 & 52027.856 & 0.013 & 1 & 106.4 & Ritter \\
\hline 11.06 .01 & 52072.266 & 0.046 & 1 & 106.5 & $\mathrm{CrAO}$ \\
\hline 13.06 .01 & 52074.272 & 0.047 & 1 & 102.8 & $\mathrm{CrAO}$ \\
\hline 14.06 .00 & 52075.271 & 0.048 & 1 & 99.5 & $\mathrm{CrAO}$ \\
\hline 06.07 .01 & 52096.828 & 0.064 & 1 & 100.2 & Ritter \\
\hline 15.07.01 & 52105.826 & 0.071 & 1 & 97.7 & Ritter \\
\hline 07.08 .01 & 52128.727 & 0.088 & 1 & 96.3 & Ritter \\
\hline 22.08 .01 & 52144.457 & 0.100 & 1 & 101.5 & Ritter \\
\hline 02.09 .01 & 52154.665 & 0.108 & 1 & 91.0 & Ritter \\
\hline 08.09.01 & 52160.496 & 0.111 & 1 & 83.9 & CFHT \\
\hline 12.09 .01 & 52164.703 & 0.115 & 1 & 87.3 & Ritter \\
\hline 17.09 .01 & 52169.659 & 0.119 & 1 & 83.3 & Ritter \\
\hline 26.10 .01 & 52209.373 & 0.148 & 3 & 82.9 & $\mathrm{CrAO}$ \\
\hline 27.10 .01 & 52210.367 & 0.149 & 1 & 83.1 & $\mathrm{CrAO}$ \\
\hline 03.11 .01 & 52216.505 & 0.154 & 1 & 83.6 & Ritter \\
\hline 02.03 .02 & 52335.517 & 0.242 & 2 & 63.1 & $\mathrm{CrAO}$ \\
\hline 07.03.02 & 52339.512 & 0.245 & 1 & 64.1 & $\mathrm{CrAO}$ \\
\hline 11.05 .02 & 52405.820 & 0.295 & 1 & 60.8 & Ritter \\
\hline 23.05 .02 & 52417.772 & 0.304 & 1 & 63.1 & Ritter \\
\hline 28.05 .02 & 52423.296 & 0.310 & 1 & 69.0 & SAO \\
\hline 07.07.02 & 52462.779 & 0.334 & 1 & 62.8 & Ritter \\
\hline 25.09 .02 & 52542.665 & 0.399 & 1 & 54.3 & $\mathrm{McD}$ \\
\hline
\end{tabular}

The observing date is presented in the form DD.MM.YY; the heliocentric Julian date (HJD) is given for the middle point of either the exposure or the whole observing time during the night (in case of multiple observations); the phase of the activity cycle is calculated according to the ephemeris of Miroshnichenko et al. (1998); $N$ is the number of spectra obtained during the night; $E W$ is the $\mathrm{H} \alpha$ line equivalent width; the observatory where the spectra were obtained is indicated in the last column.

the weather conditions. Typical exposure times were $15 \mathrm{~min}$ at CFHT and McDonald, 30 min at $\mathrm{CrAO}$ and SAO, and $1 \mathrm{~h}$ at Ritter. The $R V$ correction to the star's rest-frame was performed by assuming that the $R V$ of HD 200775 relative to the solar system is $-16 \mathrm{~km} \mathrm{~s}^{-1}$ (Pogodin et al. 2000). 
Table 2. Log of the observations in spectral regions containing photospheric lines.

\begin{tabular}{|c|c|c|c|c|c|c|c|}
\hline Date & $\begin{array}{c}\text { HJD } \\
2400000+\end{array}$ & Phase & Lines & $\begin{array}{l}R V \\
\mathrm{~km} \mathrm{~s}^{-1}\end{array}$ & $\begin{array}{c} \pm \sigma(R V) \\
\mathrm{km} \mathrm{s}^{-1}\end{array}$ & $\begin{array}{l}\text { Observa- } \\
\text { tory }\end{array}$ & $\begin{array}{l}\text { Spectro- } \\
\text { graph }\end{array}$ \\
\hline 07.06 .95 & 49875.590 & 0.40 & $\begin{array}{l}\text { He I } 5016,5048 \\
\text { S II } 5606,5647\end{array}$ & $\begin{array}{l}+4.0 \\
+5.7\end{array}$ & $\begin{array}{l}0.4 \\
2.1\end{array}$ & OHP & ÉLODIE \\
\hline 09.07 .97 & 50638.525 & 0.97 & $\begin{array}{l}\text { He I 5016, } 5048 \\
\text { S II } 5606,5647\end{array}$ & $\begin{array}{l}-8.5 \\
-7.2\end{array}$ & $\begin{array}{l}0.6 \\
0.5\end{array}$ & OHP & ÉLODIE \\
\hline 16.11 .00 & 51864.600 & 0.88 & $\begin{array}{l}\text { He I } 4009,4026 \\
\text { Si II } 4128,4131\end{array}$ & $\begin{array}{l}-21.0 \\
-9.0\end{array}$ & $\begin{array}{l}3.0 \\
1.0\end{array}$ & SAO & MSS \\
\hline 11.06 .01 & 52072.300 & 0.06 & S II 5606, 5647 & -1.4 & 0.8 & $\mathrm{SAO}$ & PFES \\
\hline 12.10 .01 & 52194.741 & 0.14 & S II 5606, 5647 & -1.6 & 1.0 & $\mathrm{McD}$ & Sandiford \\
\hline 29.11 .01 & 52242.647 & 0.17 & S II 5606, 5647 & +2.9 & 0.2 & $\mathrm{SAO}$ & LYNX \\
\hline 06.12 .01 & 52250.300 & 0.17 & $\begin{array}{l}\text { He I } 4009,4026 \\
\text { Si II } 4128,4131\end{array}$ & $\begin{array}{l}+15.0 \\
+17.0\end{array}$ & $\begin{array}{l}3.0 \\
5.0\end{array}$ & $\mathrm{SAO}$ & MSS \\
\hline 27.04 .02 & 52391.510 & 0.28 & $\begin{array}{l}\text { He I } 5016,5048 \\
\text { S II } 5606,5647\end{array}$ & $\begin{array}{l}-3.0 \\
-4.7\end{array}$ & $\begin{array}{l}0.2 \\
0.8\end{array}$ & $\mathrm{SAO}$ & NES \\
\hline 28.05 .02 & 52423.296 & 0.31 & S II 5606, 5647 & -1.7 & 3.0 & $\mathrm{SAO}$ & LYNX \\
\hline 12.07 .02 & 52467.500 & 0.33 & He I 4009, 4026 & +4.0 & 2.0 & CFHT & Gecko \\
\hline 26.07 .02 & 52482.233 & 0.34 & $\begin{array}{l}\text { He I 5016, } 5048 \\
\text { S II } 5606,5647\end{array}$ & $\begin{array}{l}-4.2 \\
-0.6\end{array}$ & $\begin{array}{l}0.8 \\
0.7\end{array}$ & $\mathrm{SAO}$ & NES \\
\hline 25.09 .02 & 52542.665 & 0.40 & $\begin{array}{l}\text { S II } 5606,5647 \\
\text { S II } 5453\end{array}$ & -2.1 & 3.0 & $\mathrm{McD}$ & Sandiford \\
\hline
\end{tabular}

The observational data are presented in the following columns: (1) - the observing date (DD.MM.YY); (2) - the heliocentric Julian date; (3) - the phase of the $1345^{d}$-period of activity calculated according to the ephemeris of Miroshnichenko et al. (1998); (4) - the photospheric lines whose radial velocities were measured; (5) and (6) - the mean radial velocity for a given element and its error, respectively; (7) - the observatory where the data were obtained; $(8)$ - the spectrograph which was used to obtain the spectrum.

\section{Results}

\subsection{The $\mathrm{H} \alpha$ line}

The epoch of maximum $\mathrm{H} \alpha$ equivalent width $(E W)$, zero phase $(\phi)$ in the following, and the period of its cyclic variability (1345 days) found by Miroshnichenko et al. (1998), predicts the next active phase of HD 200775 in April 2001.

We started the new observing run in September 2000 at a phase $\phi \sim 0.85$, when the $\mathrm{H} \alpha$ line quickly strengthened (see Table 1). Thus even at the beginning of the run, signs of the expected forthcoming active event were clearly seen. The occurrence and character of the active phenomena in 2001 were similar to those that occurred in 1997 (Pogodin et al. 2000).

Figure 1 shows the $\mathrm{H} \alpha$ profile structure change in 2000-2002 in comparison with that observed in 1997-1998, before, during, and after the previous period of high activity at approximately the same phases. In December $2000(\phi=0.90)$ the $E W$ was larger than in the previous low state by $\sim 50 \%$, but the profile shape had not begun changing yet. As in the low-activity state, the line had a double-peaked emission profile with the blue peak stronger. This is in contrast the profile evolution in 1997, when the beginning of the profile transformation was clearly seen already at $\phi=0.90$ (Fig. 1, left panel).
At $\phi=0.97-0.98$ and $\phi=0.14-0.17$, the profile changes were the same in 1997-1998 and in 2000-2002. Instead of a single absorption component located at a small positive velocity between the two emission peaks, two absorptions of a smaller depth were formed at negative and positive velocities before $\phi=0$. Later the velocity shift of the blue absorption feature quickly decreased and reached $\sim 0 \mathrm{~km} \mathrm{~s}^{-1}$ at $\phi=0$, while the red feature remained at the same position for a few more months. At $\phi=0.15$ both features were still distinguishable, but partly merged together. At $\phi=0.30$ the usual doublepeaked emission profile, similar to that at the $\phi=0.90$, was observed again. It is worth noting that during the previous activity cycle in 1997-1998 the $\mathrm{H} \alpha$ profile returned to this usual type at a later phase. One can conclude that the active period in 2001 lasted for a shorter time than in 1997.

In more details, common and distinctive characteristics of the active phenomena seen in the $\mathrm{H} \alpha$ profile in 1997 and in 2001 are illustrated in Fig. 2 where our 1997 data, published in Pogodin et al. (2000), are used for comparison. It is clearly seen, that the timescale of the $E W$ rise and subsequent decrease in 2001 is significantly shorter than the same timescale in 1997. Before the 2001 maximum, the $E W$ curve demonstrated a pronounced jump. The same feature may have been 


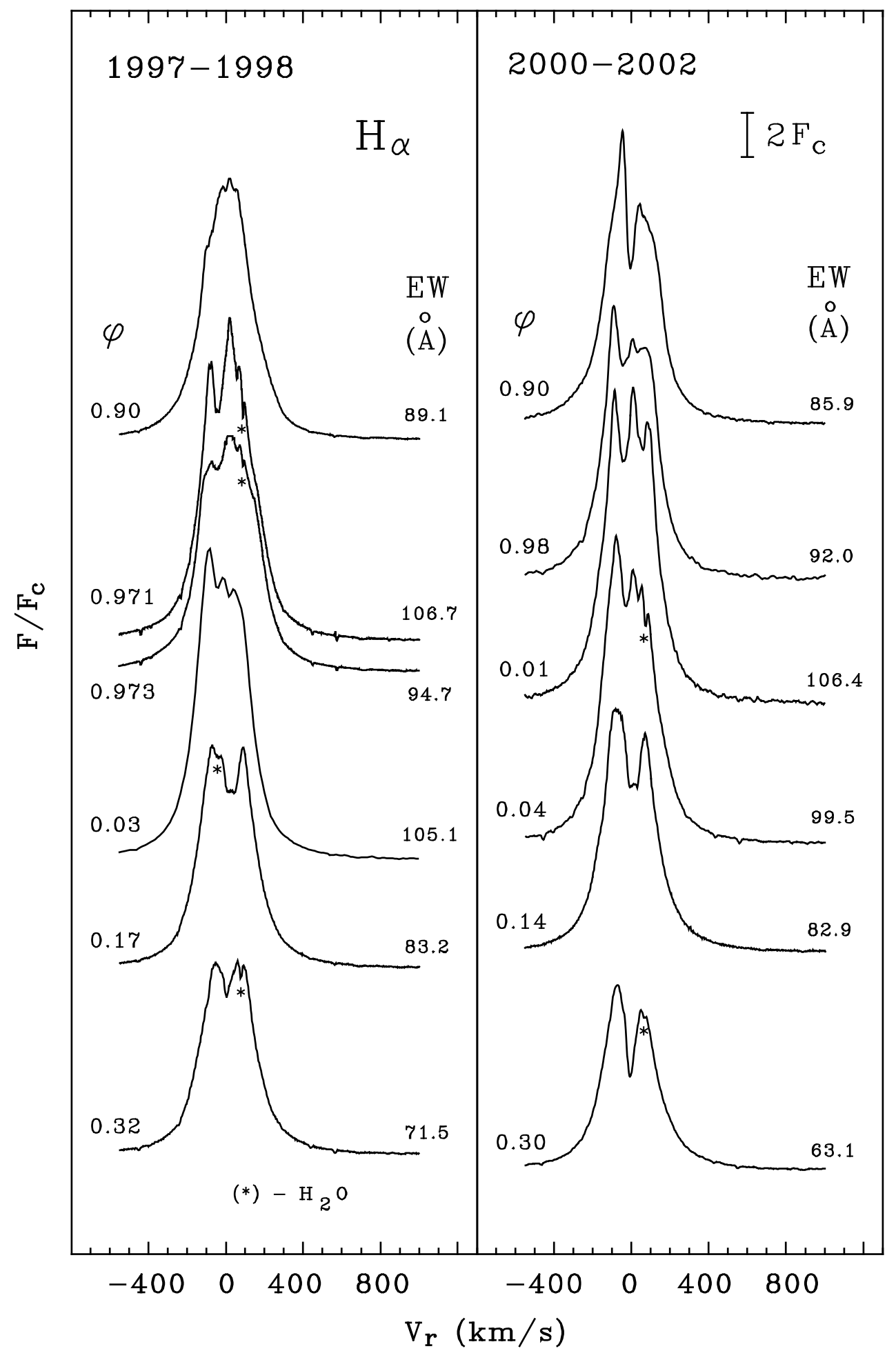

Fig. 1. Typical $\mathrm{H} \alpha$ profiles normalized to the continuum $\left(F_{\mathrm{c}}\right)$ illustrating the evolution of the HD 200775 spectrum in 2000-2002 (right panel) in comparison with those observed in 1997-1998 (left panel) at similar phases $\phi$. The water vapor lines near the top of the emission peak are marked by the ( $\star$ ) symbols. The $R V$ scale is given with respect to the star.

present in 1997, observations are too sparse to support a definite conclusion.

The temporal behaviour of the central absorption features in the $\mathrm{H} \alpha$ emission profile is illustrated in the middle panel of Fig. 2. Qualitatively, the character of the evolution of these features near the 2001 active phase is similar to that observed in 1997 , except the $R V \mathrm{~s}$ of both the blue and the red features in 2001 are $20-30 \mathrm{~km} \mathrm{~s}^{-1}$ larger than in 1997.

The bottom panel of Fig. 2 shows the evolution of the bisector velocity, $V_{\text {bis }}$, determined at intensity levels of 1.5 to 2.0 times the continuum (see Sect. 3.3). One can see that the variations are similar in 1997 and in 2001 . The $V_{\text {bis }}$ shows a 


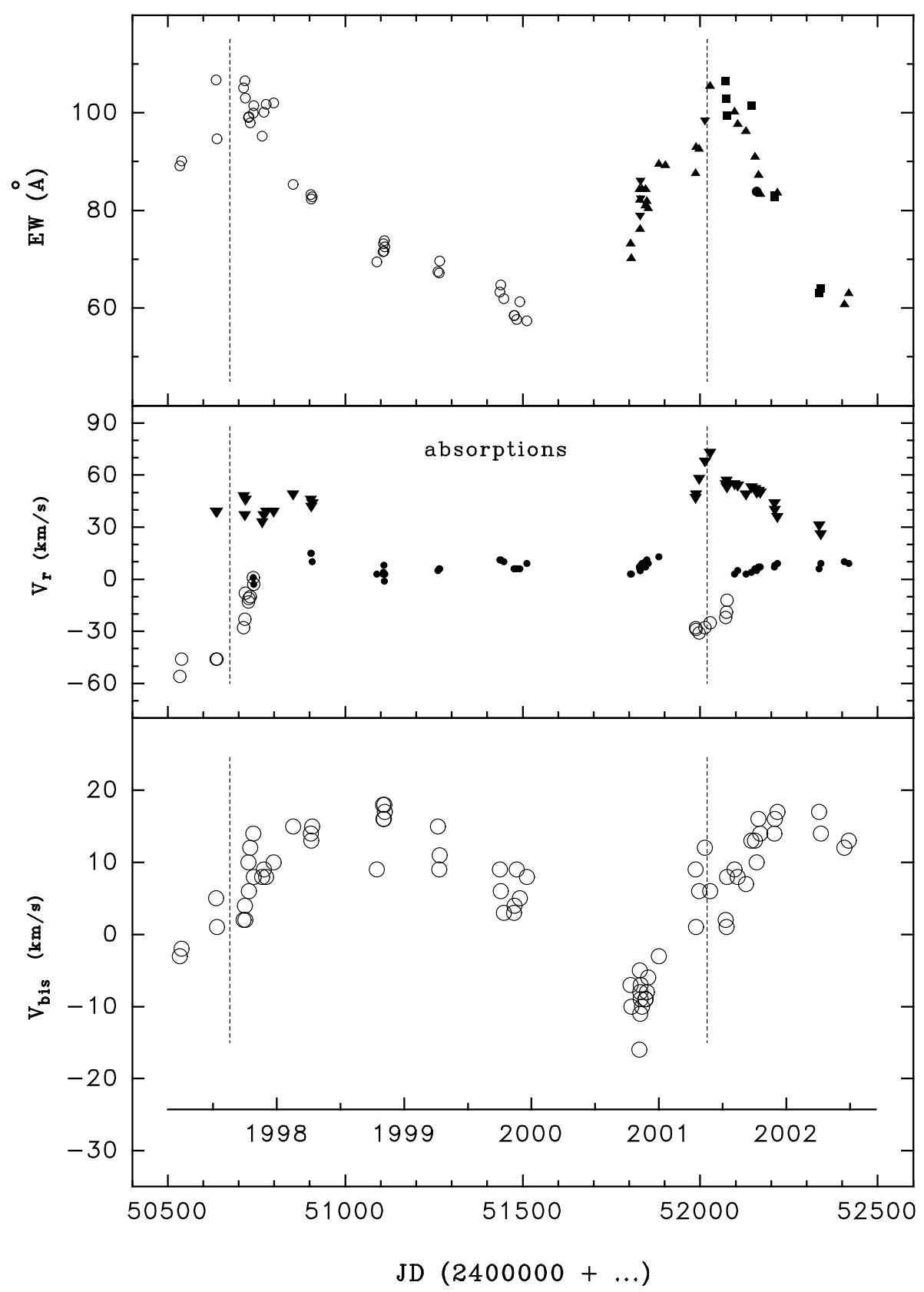

Fig. 2. Temporal behaviour of the $\mathrm{H} \alpha$ line variations in the spectrum of HD 200775 in 1997-2002. The $E W$ variations are shown in the upper panel. In the middle panel the circles, triangles and dots illustrate $R V \mathrm{~s}$ of the blueshifted, redshifted, and central absorption features, respectively. The lower panel presents the variations of the bisector velocity $V_{\text {bis }}$ derived at intensity of levels of $1.5-2.0 F_{\mathrm{c}}$. The dashed vertical lines show the $E W$ maximum epochs in 1997 and 2001 according to the ephemeris by Miroshnichenko et al. (1998). The measurement uncertainites are as follows: $1-2 \AA$ for the $E W \mathrm{~s}, 1-2 \mathrm{~km} \mathrm{~s}^{-1}$ for the absorption feature $R V \mathrm{~s}$ and $3 \mathrm{~km} \mathrm{~s}^{-1}$ for $V_{\text {bis }}$ at $1.5-2.0 F_{\mathrm{c}}$.

deep minimum (down to $-15 \mathrm{~km} \mathrm{~s}^{-1}$ ) at $\phi \sim 0.85$, a steady rise, and a flat maximum $\left(+15 \mathrm{~km} \mathrm{~s}^{-1}\right) \phi=0.2-0.3$.

We can draw the following conclusions from our spectroscopic results: a) the behaviour of the $\mathrm{H} \alpha E W$ is consistent with periodicity with $P=1345$ days; b) the general picture of the $\mathrm{H} \alpha$ profile evolution during both periods of high activity in 1997 and 2001 is qualitatively similar; and c) differences in the duration of the active phase and in the behaviour of the absorption features were observed during these two periods.

\subsection{Photospheric lines}

One of the most important signs of binarity is a regular positional variability of photospheric lines at different phases of the expected orbital period. This is why we included in our programme a spectroscopic investigation of HD 200775 in the regions of photospheric lines of He I, Si II, and S II, which are not contaminated by blending or affected by the envelope emission (see Table 2).

The $R V$ of the weak $\mathrm{Si}$ II and $\mathrm{S}$ II lines was determined as the centroid of the photospheric profile. For the stronger 


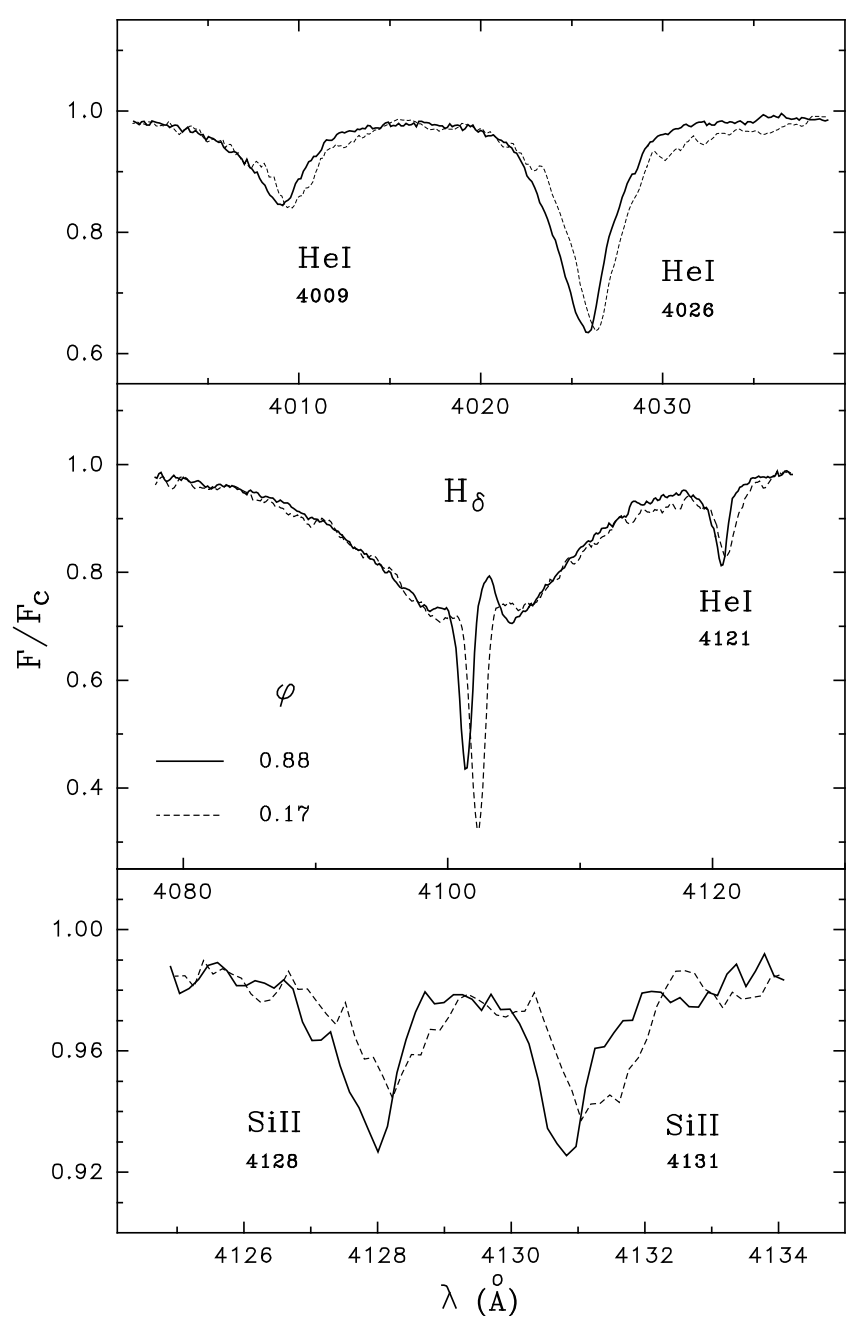

Fig. 3. Positional variations of strong photospheric lines in the spectrum of HD 200775. The SAO spectra obtained at $\phi=0.88$ and $\phi=0.17$ are shown by the solid and dashed lines, respectively. The intensity is normalized to the underlying continuum, while the wavelengths are heliocentric.

He I lines the same procedure was used, but only for the central part of the profile where $F / F_{\mathrm{c}} \leq 0.5$. The velocity errors were derived as deviations of the individual measurements from the mean velocity for a given element and date.

Figure 3 shows our data for the stronger photospheric lines in the blue spectral region obtained before and after the 2001 maximum. The wavelength shift of the He I $\lambda \lambda$ 4009, 4026, and $4121 \AA$ lines and the Si II $\lambda \lambda 4126$ and $4131 \AA$ lines at different $\phi$ is clearly seen. The profiles observed at $\phi=0.17$ are shifted redward in the object's rest frame by $+15 \pm 3 \mathrm{~km} \mathrm{~s}^{-1}$ for the $\mathrm{He} \mathrm{I}$ lines and by $+17 \pm 5 \mathrm{~km} \mathrm{~s}^{-1}$ for the Si II lines. An additional wide depression with respect to the profiles at $\phi=0.88$ is present in the red wing of the He I $\lambda \lambda 4009$ and $4026 \AA$ lines and the $\mathrm{H} \delta$ line. Some local features are also seen in the blue wing of the He I lines. At $\phi=0.88$ the positions of these lines are $-21 \pm 3 \mathrm{~km} \mathrm{~s}^{-1}$ (for He I) and $-9 \pm 1 \mathrm{~km} \mathrm{~s}^{-1}$ (for Si II). We note here that except for the appearance of the red depression at $\phi=0.17$, the photospheric wings in $\mathrm{H} \delta$ show no positional change at other phases. Only the CS doublepeaked emission component located in the centre of the wide

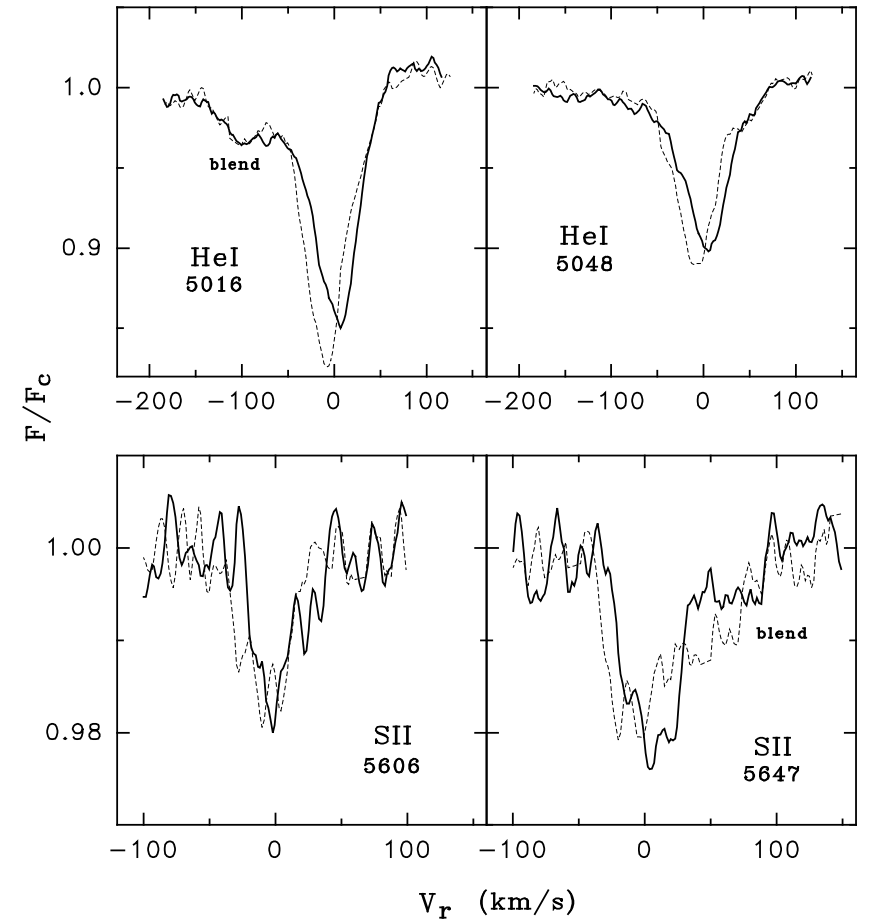

Fig. 4. Profiles of the He I and the weak S II lines in the spectrum of HD 200775 obtained on June 7, $1995(\phi=0.40)$ and on July 9, 1997 $(\phi=0.97)$ marked by the solid and dashed lines, respectively. The velocity scale is given with respect to the star.

photospheric profile is significantly variable. A similar demonstration of the positional variability of the weaker photospheric lines (He I 5015.67, 5047.74 $\AA$ and S II 5606.11, 5646.98 $\AA$ ) is presented in Fig. 4.

The variations of the stronger lines seen in the best quality spectra suggest that the velocity position is not the only variable parameter. The line profiles also undergo significant changes. The examples include the local features, which are formed at some phases on the wide absorption wings of the He I 4009, 4026 lines (see Fig. 3), and pronounced variations of the depth of the He I 5016, $5048 \AA$ (Fig. 4) and Si II 4128, $4134 \AA$ (Fig. 3) lines. The variations may be due to the influence of the CS envelope. We can assume that the positional stability of the $\mathrm{H} \delta$ line wings illustrated in Fig. 3 is an apparent effect connected with weak CS emission wings on top of the photospheric wings, which are positionally shifted in the same direction as the CS ones. Nevertheless, as clearly seen in Figs. 3 and 4, the positional change of the photospheric lines is the dominant type of variability, and this change is detected in both strong and weak lines. We conclude that the behaviour of the photospheric lines is consistent with the suggestion of binarity.

\subsection{The orbital solutions}

With additional evidence of binarity obtained, the next step of our investigation is to derive properties of the companions and orbital elements from the $R V$ curve. So far we do not have enough data for the photospheric lines to construct such a curve with satisfactory accuracy. Furthermore, the line profiles are 


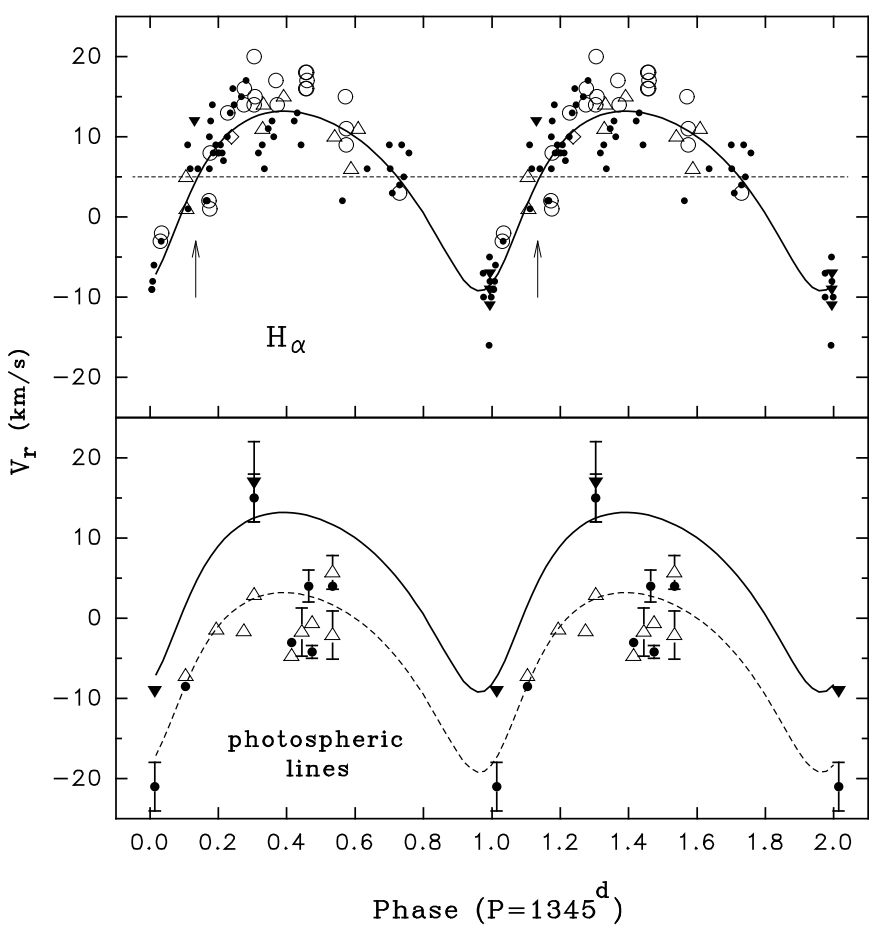

Fig. 5. Top: the bisector velocity $V_{\text {bis }}$ derived at the level $1.5-2.0 F_{\mathrm{c}}$ folded with the period $P=1345$ days. The least-squares fit to the observations indicated by the solid line illustrates the calculated $R V$ curve. The initial phase corresponds to the epoch of periastron passage. The phase when the observed $E W$ of the $\mathrm{H} \alpha$ emission is at maximum is indicated by the arrows. The dashed line corresponds to the $\gamma$-velocity. Data obtained at different observatories are marked by following symbols: filled circles - Ritter; open circles - CrAO; upward open triangles - OHP; downward filled triangles - SAO; open diamands - CFHT. Bottom: the $R V$ of the photospheric lines in comparison with the curve calculated for the $\mathrm{H} \alpha$ data. The following symbols mark the $R V$ of lines for different elements: filled circles - He I, downward filled triangles - Si II, and upward open triangles - S II. The dashed line indicate the $R V$ curve for the $\mathrm{H} \alpha$ data but shifted on $-9 \mathrm{~km} \mathrm{~s}^{-1}$.

not stable and can be affected by a time-variable contribution from the CS matter that can lead to an incorrect orbital solution. However, we think it is reasonable to make preliminary estimates of the orbital and companions' parameters using our data for the $\mathrm{H} \alpha$ line, which originates in the CS envelope. This approach has already been applied to determine orbital elements of some binary Be stars (e.g., Božić et al. 1995; Harmanec et al. $2000)$. It assumes that the $R V$ curve based on the outer wings of the Balmer emission lines, which are formed in the inner axially symmetric parts of the gaseous disk, reflect the true orbital motion of the central star. We suggest that the bisector velocity of the $\mathrm{H} \alpha$ profile of $\mathrm{HD} 200775$ defined at a level 1.5-2.0 $F_{\mathrm{c}}$ closely follows the true $R V$ of the primary.

We used all our measurements of $V_{\text {bis }}$ obtained during the 2000-2002 observing run as well as our previous data collected in 1994-1999 (Pogodin et al. 2000) to construct a phase curve covering two periods of activity ( $~ 90$ measurements). The least-squares method was applied to derive the orbital elements. From this, we found the following parameters: $P=$ $1341 \pm 23$ days; the periastron epoch $T_{\mathrm{o}}=\mathrm{JD} 2449149 \pm 87$; $a_{1} \sin i=284 \pm 18 R_{\odot}$, where $a_{1}$ is the semi-major axis of the primary companion and $i$ is the inclination angle of the orbit to the line of sight; the orbital eccentricity $e=0.29 \pm 0.07$; the periastron longitude $\omega=203 \pm 22^{\circ}$; the semi-amplitude of the cyclic $R V$ variations $K=11.2 \pm 0.7 \mathrm{~km} \mathrm{~s}^{-1}$; the $R V$ of the system mass centre $\gamma=5.0 \pm 0.6 \mathrm{~km} \mathrm{~s}^{-1}$; and the mass function for the secondary companion $\mathrm{f}\left(M_{2}\right)=0.175 \pm 0.035 M_{\odot}$.

We note that the different $\gamma$ values obtained at different instruments do not significantly exceed the error levels: $4.14 \pm 0.65 \mathrm{~km} \mathrm{~s}^{-1}$ (Ritter); $4.25 \pm 1.25 \mathrm{~km} \mathrm{~s}^{-1}$ (OHP); $6.01 \pm 2.26 \mathrm{~km} \mathrm{~s}^{-1}(\mathrm{SAO})$ and $7.24 \pm 1.21 \mathrm{~km} \mathrm{~s}^{-1}(\mathrm{CrAO})$. Test calculations, carried out with the individual values of $\gamma$ for different instruments, show that their differences practically do not change the obtained orbital solution.

It is remarkable that the derived orbital period is in excellent agreement with that derived by Miroshnichenko et al. (1998) on the basis of the $\mathrm{H} \alpha E W$ variability. We use the 1345-day period based on the $E W$ variations for the phase $R V$ curves, because it is determined with a better accuracy (2 days) than the one found from the $R V$ variations. The data points corresponding to the photospheric lines demonstrate approximately the same phase dependence, except they are shifted by $-9 \mathrm{~km} \mathrm{~s}^{-1}$ with respect to the $\mathrm{H} \alpha R V$ curve (Fig. 5, bottom). Despite the orbital solution for the photospheric data is much less precise, it leads to the values for $T_{0}$ (JD2 $449162 \pm$ 234 days) and $\omega\left(224 \pm 11^{\circ}\right)$, close to those derived from the $\mathrm{H} \alpha$ data. The different value of $\gamma=-4.1 \pm 2.7 \mathrm{~km} \mathrm{~s}^{-1}$ $\left(-4.4 \pm 2.8 \mathrm{~km} \mathrm{~s}^{-1}\right.$ for the He I lines and $-3.3 \pm 2.6 \mathrm{~km} \mathrm{~s}^{-1}$ for the S II lines) may be due to a rather small number of measurements. Another reason for the differences between the $\mathrm{H} \alpha$ and photospheric solutions is possible variations of the photospheric line profiles. The individual data points show a large dispersion, which significantly exceeds the measurement errors as well as the expected variations due to the orbital motion. A refinement of both (photospheric and $\mathrm{H} \alpha$ ) $R V$ curves on the basis of new observations and its interpretation is the main task of our forthcoming investigation of HD 200775.

\section{Discussion}

\subsection{Nature of the components}

As stated above, the temporal behaviour of the $E W(\mathrm{H} \alpha), R V$ of the local $\mathrm{H} \alpha$ components, and $V_{\text {bis }}$ of the $\mathrm{H} \alpha$ emission profile shows that the cyclic large-amplitude variations with the period $P=1345$ days is the main type of variability observed in HD 200775 in 1994-2002. The period and the shape of the velocity curve are consistent with a binary model for the object. Additionally, positional and intensity changes at shorter time scales are likely to be present in photospheric lines. These variations make the $R V$ phase curve due to the orbital motion more uncertain. Nevertheless, we can use our preliminary orbital solution based on the $\mathrm{H} \alpha$ data (because that for the photospheric line data is much less precise) to estimate some parameters of the system and its components. 
Assuming that the primary's mass $M_{1}$ and radius $R_{1}$ are $10 M_{\odot}$ and $8 R_{\odot}$, respectively, (see Hillenbrand et al. 1992; Böhm \& Catala 1995; van den Ancker et al. 1998 and references therein) we can estimate a mass of the secondary companion and a distance between the companions using an estimate for the orbital inclination angle $i$. Watt et al. (1986) showed that HD 200775 is surrounded by an extended CO disk with the axis tilted at an angle of $70^{\circ}$ to the line-of-sight. We can assume that the orbital plane coincides with the equatorial plane of the $\mathrm{CO}$ disk.

Thus, the mass function $f\left(M_{2}\right)=0.175 \pm 0.035 M_{\odot}$ derived from the orbital solution and $i=70^{\circ}$ give the secondary's mass $M_{2}=3.35 \pm 0.25 M_{\odot}$ and the semi-major axis of the primary $\left(a_{1}=30 R_{1}\right)$ and that of the secondary $\left(a_{2}=90 R_{1}\right)$. The distance between the stars must be $\sim 80 R_{1}$ at periastron and about $160 R_{1}$ at apoastron. Even when the stars pass through periastron, the distance between them is too large for the system to be an interacting binary.

The secondary's physical characteristics strongly depend on the system's age. At present there is no way to determine the age precisely, because a star with $M_{1} \approx 10 M_{\odot}$ only emerges from its optically-thick protostellar cocoon very close to the main sequence, and has virtually no PMS evolutionary phase at all. If the age of the system is $\sim 10^{6}$ years, the secondary is a late B-type star, but if the age of the system is $\leq 10^{5}$ years, the secondary is a G-type giant star of 3 to 10 times lower luminosity (Palla \& Stahler 1993). If the secondary is a late-type star, its continuum visual flux is expected to be 20-30 times smaller than that from the primary. Thus, it is difficult to find its features in the observed spectrum against the bright background of the primary. If the secondary is older than $\sim 10^{5}$ years, it is $\sim 10$ times fainter than the primary, and its spectral signature should be detectable in high $S / N$ high-resolution spectra.

\subsection{Possible origin of active phenomena in the CS envelope}

In the previous sections, it was shown that the likely periodic character of the $\mathrm{H} \alpha$ line variability, the presence of $R V$ variations in the photospheric lines, and the existence of a selfconsistent orbital solution for both $\mathrm{H} \alpha$ and the photospheric lines make it possible that HD 200775 is a binary.

Our analysis of the light curve of HD 200775 in the $U B V R$ bands taken from the data base of Herbst et al. (1994) shows that the object was $\sim 0$. 04 brighter in the $V$-band during the active phases in July 1986 and December 1993, as it has been noted by Miroshnichenko et al. (1998). At the same time, during other active phases in April 1990 and August 1997 its brightness was the same as during the corresponding minima of activity. Thus, we cannot conclude that the visual brightness of HD 200775 is correlated with its $E W(\mathrm{H} \alpha)$.

We assume that, like the primary companion, the secondary of the young binary system is surrounded, by an extended CS envelope, which is usual for a PMS star. It seems reasonable that the variability in the $E W$ of $\mathrm{H} \alpha$ is caused by interaction between the CS envelopes of the two stars, since the $\mathrm{H} \alpha E W$ has a maximum near periastron. However, the maximum might be shifted from periastron epoch if the orbital plane and the companions' disks symmetry planes do not coincide. Detailed discussion of this process is beyond the scope of this paper, because the available evidence about the nature of the secondary is very sparse, and models for the interaction between two CS envelopes have not been developed. Nevertheless, it is reasonable to conjecture that the rise in the $\mathrm{H} \alpha$ strength near periastron is caused by mutual heating of the two stars' envelopes as they draw together. In any case, the actual mechanisms of the object's activity remain to be revealed in a forthcoming investigation on the basis of additional spectroscopic data.

In a study similar to ours, Ismailov (2003) concluded that HD 200775 is a spectroscopic binary with a period $P=1180 \pm$ 60 days and an eccentricity $e=0.10 \pm 0.01$, quite different from the values derived in the present study. Those results are based mainly on the radial velocities of He I $\lambda 5876 \AA$. More than half of the data used for the frequency analysis ( 40 out of 71 points) were taken from our previous paper (Pogodin et al. 2000). In that paper we noted that the profile of this He I line is very complex, and its central absorption is strongly affected by the CS emission. Actually, the $R V$ of the absorption shows a large-amplitude variability on a time-scale of about 1000 days. However, it cannot be used as an indicator of the orbital motion of the star. This variability is not correlated with the change of the emission in $\mathrm{H} \alpha$, and its origin is yet to be explained.

Our principal concern is that Ismailov (2003) found substantial variability in the $E W$ of $\mathrm{H} \alpha$ during the course of individual nights, including changes as large as $20 \AA$, which our observations do not confirm. As seen in Table 1, our $\mathrm{H} \alpha E W \mathrm{~s}$ measured in the spectra obtained on consequtive nights even with different instruments do not differ more than $\sim 4 \AA$. The difference is less than $2 \AA$ for the data obtained during the same night with the same instrument. Therefore, we conclude that Ismailov's observational material may be subject to significant, unrecognized instabilities of instrumental origin, and it would be premature to view them as contradictory to ours.

The frequency analysis of the low-amplitude photometric variability of HD 200775 by Ismailov (2003) is too cursory. It is not clear whether the brightness variations follow the phase of the periodic spectral variations or the effect is accidental, because the amplitude of the photometric variations does not exceed the errors. In order to draw a more definite conclusion, the entire temporal series (spanning nearly 15 years) can be split into two or more groups which have to be analysed separately. If the photometric variability is due to eclipses, the orbital period will show up in each of the groups. We do not find any significant correlation between the object's brightness in the $V$-band and its phase of activity (see the beginning of this subsection).

\section{Conclusions}

We presented new results of our spectroscopic observations of the Herbig Be star HD 200775 obtained in 2000-2002. The following phenomena, similar to those observed in 1997, were observed in 2001:

- the increase of the $\mathrm{H} \alpha$ emission with a maximum in the middle of 2001, and its a consequent decrease; 
- the appearance and temporal variations of weak blueand red-shifted absorption components in the $\mathrm{H} \alpha$ profile, formed instead of the single deep central absorption separating two emission peaks usually observed during lowactivity states of HD 200775;

- the variations of the $\mathrm{H} \alpha$ profile asymmetry during the active phases.

The analysis of the data allows us to make the following conclusions:

1. The occurrence of a new active phase in the object's spectral behaviour in 2001 confirmed the cyclic character with $P=1345$ days predicted by Miroshnichenko et al. (1998).

2. A noticeable change of the $R V$ in the He I and Si II photospheric lines before and after the activity maximum was detected.

3. We suggest that the active phenomena in the spectral behaviour of HD 200775 are influenced by the binary nature of the object.

4. The $R V$ curves derived from our $\mathrm{H} \alpha$ data obtained in 1994-2002 were used to determine preliminary orbital elements and stellar parameters. We suggest that neither companion comes close to filling its Roche lobe. The system consists of a B2e primary star and a pre-main sequence secondary of a $M \approx 3.5 M_{\odot}$ with a distance $\sim 1000 R_{\odot}$ between them. The orbital eccentricity $e \sim 0.3$.

5. Our observational data are as yet inadequate to reconstruct the structure of the CS medium around HD 200775 and to identify actual mechanisms for the activity. These tasks remain to be solved in our future study of this unique object.

We would like to emphasize that the following investigations can provide a useful guide for further understanding the properties of the system and its components:

- frequent high-resolution and high- $S / N$ spectroscopy is needed to search for the secondary;

- measurements of the $R V$ of photospheric lines at different phases will allow refinement of the orbital solution;

- interferometric observations during the object's low activity states, when the companions are at maximum separation (about 20 marcsec), might reveal the secondary companion in a direct image.

The first results of near-IR speckle interferometry (Millan-Gabet et al. 2001) have already shown the presence of a companion of HD 200775 at a distance of $20 \pm 6$ marcsec from the object, but this discovery has to be confirmed.
Acknowledgements. We thank $\mathrm{V}$. El'kin for providing us with additional spectroscopic data obtained at the Special Astrophysical Observatory (Russia) in October 2000. Anatoly Miroshnichenko and Karen Bjorkman acknowledge support from NASA grant NAG5-8054. Karen Bjorkman is a Cottrell Scholar of the Research Corporation, and gratefully acknowledges their support. Support for observational research at Ritter Observatory is provided by The University of Toledo, by NSF grant AST-9024802 to B. W. Bopp, and by a grant from the Fund for Astrophysical Research. Technical support is provided by R. J. Burmeister.

\section{References}

van den Ancker, M. E., de Winter, D., \& Tjin A Djie, H. R. E. 1998, A\&A, 330, 145

Baudrand, J., \& Vitry, R. 2000, in Proc. SPIE, 4008, 182

Baudrand, J., \& Walker, G. A. H. 2001, PASP, 113, 851

Beskrovnaya, N. G., Pogodin, M. A., Shcherbakov, A. G., \& Tarasov, A. E. 1994, A\&A, 287, 564

Božić, H., Harmanec, P., Horn, J., et al. 1995, A\&A, 304, 235

Böhm, T., \& Catala, C. 1995, A\&A, 301, 155

Corporon, P., \& Lagrange, A.-M. 1999, A\&AS, 136, 429

Harmanec, P., Habuda, P., Štefl, S., et al. 2000, A\&A, 364, L85

Herbst, W., Herbst, D. K., Grossman, E. J., \& Weinstein, D. 1994, AJ, 108, 1906

Hillenbrand, L. A., Strom, S. E., Vrba, F. J., \& Keene, J. 1992, ApJ, 397, 613

Ismailov, N. Z. 2003, A. Rep., 47, 206

McCarthy, J. K., Sandiford, B. A., Boyd, D., \& Booth, J. 1993, PASP, 105,881

Millan-Gabet, R. S., Shloerb, F. P., \& Traub, W. A. 2001, ApJ, 546, 358

Miroshnichenko, A. S., Mulliss, C. L., Bjorkman, K. S., et al. 1998, PASP, 110, 883

Palla, F., \& Stahler, S. W. 1993, ApJ, 418, 414

Panchuk, V. E., Najdenov, I. D., Klochkova, V. G., et al. 1998, Bull. Special Astrophys. Observ., 44, 127

Panchuk, V. E., Klochkova, V. G., Najdenov, I. D., et al. 1999a, Preprint of the Special Astrophys. Observ., 139

Panchuk, V. E., Klochkova, V. G., \& Najdenov, I. D. 1999b, Preprint of the Special Astrophys. Observ., 135

Pogodin, M. A., Miroshnichenko, A. S., Bjorkman, K. S., et al. 2000, A\&A, 359, 299

Watt, G. D., Burton, W. B., Choe, S.-U., \& List, H. S. 1986, A\&A, 163,194 